\section{Pericardial tamponade and pancytopenia as the first manifestation of mixed connective tissue disorder and its complete reversal with corticosteroids}

\author{
Ankur Jain \\ Department of Medicine, Maulana Azad \\ Medical College, New Delhi, India
}

\begin{abstract}
We report a case of a 25-year-old lady who presented to our department with complaints of easy fatigability and shortness of breath since one week. She had a history of Raynaud's phenomenon. Examination revealed scleroderma like skin changes and pericardial friction rub. Investigations revealed high titer of anti-U1 RNP antibodies along with co-existing pancytopenia. Chest x-ray and echocardiography confirmed pericardial tamponade. Patient was diagnosed as having mixed connective tissue disorder (MCTD) and she was started on high dose prednisolone, which led to complete reversal of pancytopenia and pericardial tamponade after 1 month of treatment. There are only 6 reported cases of pericardial tamponade in a patient with MCTD, and none of them had pancytopenia. Present case highlights the need to investigate the patient of pericardial tamponade for MCTD, especially in the presence of pancytopenia and relevant clinical history, as prompt treatment with corticosteroids can avoid invasive procedures like pericardiocentesis.
\end{abstract}

\section{Case Report}

We report a case of a 25-year old lady who presented to our OPD with the complaints of generalized body swelling since one month and shortness of breath since one week. Patient was well one month back when she started developing swelling over the body which she noticed first on face and gradually progressed to involve the whole body which was associated with the simultaneous thickening of the overlying skin and appearance of depigmented lesions over the hands and back. She developed increasing difficulty in breathing since the last one week which was associated with fever and chest pain for which she presented to our OPD. Patient was a married woman and had history of two consecutive abortions in the past and had no live issues. She had a 1 year history of Raynaud's phenom- enon. There was no history of oral ulcers, arthralgias, arthritis, photosensitivity, facial erythema, jaundice, malena, hemetemesis, reduced urine output, hematuria and family history of autoimmune disease. On examination, patient was conscious and oriented to time, place and person and was afebrile to touch. Her BP was 110/60 mmHg and pulse rate was $108 / \mathrm{min}$. General physical examination was remarkable for the presence of pallor, but there was no icterus, cyanosis, clubbing or lymphadenopathy. Patient's JVP was raised and she had bilateral pitting type of pedal edema and periorbital edema. There was thickening and diffuse pigmentation of skin over both the limbs, with perifollicular depigmented macules seen over the arms and back, nonscarring alopecia, and reduced mouth opening (Figure 1). Dorsum of bilateral hands had puffy appearance with loss of wrinkles and dermal appendages. Cardiovascular examination revealed the presence of pericardial friction rub. Respiratory, abdominal and central nervous system examination were unremarkable.

Patient's routine investigations revealed: $\mathrm{Hb} 78 \mathrm{gm} / \mathrm{L}$; total leucocyte count $3700 \times 10^{9} / \mathrm{L}$; differential count; $52 \%$ polymorphs; $41 \%$ lymphocytes; $4 \%$ eosinophils; $3 \%$ monocytes; platelet counts $40 \times 10^{9} / \mathrm{L}$. Peripheral smear revealed pancytopenia with normocytic and normochromic anemia. Erythrocyte sedimentation rate $65 \mathrm{~mm} / \mathrm{hr}$, liver and kidney function tests were normal, total calcium $1.92 \mathrm{mmol} / \mathrm{L}$, phosphorous $1.55 \mathrm{mmol} / \mathrm{L}$, total proteins 48 $\mathrm{gm} / \mathrm{L}$, serum albumin $15 \mathrm{gm} / \mathrm{L}$, total cholesterol $5.62 \mathrm{mmol} / \mathrm{L}$, serum triglycerides $4.8 \mathrm{mmol} / \mathrm{L}$. Urine analysis revealed the presence of 4+ proteinuria by dipstick and 24 hour urinary protein was $3100 \mathrm{mg} / 24$ hours. Urine examination revealed inactive sediment and there were no red cell casts, crystals or pus cells. Renal biopsy of the patient was done which revealed membranous nephropathy. Patient's ANA was positive (1:256, speckled positivity), anti-double stranded DNA $5.8 \mathrm{IU} / \mathrm{mL}$ (normal $<30$ $\mathrm{IU} / \mathrm{mL}$ ), anti-Sm antigen, anti-Scl 70, anti-centromere, anti-SSB, P-ANCA and C-ANCA were negative. Patient's sera tested positive for anti-U1RNP (1:256) and anti-SSA/Ro-52 (1:180). Patient's HIV-1 and HIV-2 serology, hepatitis B surface antigen and anti-HCV were negative. Patient's coomb's test, antiphospholipid antibodies and D-Dimers were negative. Patient's coagulation profile and fibrinogen levels were normal. Her TSH $8.92 \mathrm{mIU} / \mathrm{mL}$, FT4 $9.04 \mathrm{pmol} / \mathrm{L}, \mathrm{FT} 30.025 \mathrm{pmol} / \mathrm{L}$ (suggestive of subclinical hypothyroidism). Rest of the hormone profile (FSH, LH, prolactin, cortisol) was normal. Patient's skin biopsy was done which revealed basket weave hyperkeratosis, prominent basal layer, perivascular edema in dermis with chronic inflammation. Dermis was homogenous with dermal fibrosis confirmed by masson's trichrome stain with changes con-
Correspondence: Ankur Jain, Department of Medicine, Maulana Azad Medical College, Bahadur Shah Zafar Marg, New Delhi, DL 110002 , India.

Tel.: +91.011.273.25045

E-mail: drankur589@yahoo.in

Key words: mixed connective tissue disorder, pericardial tamponade, pancytopenia.

Conflict of interests: the authors declare no potential conflict of interests.

Received for publication: 19 April 2014.

Revision received: 10 May 2014.

Accepted for publication: 29 May 2014.

This work is licensed under a Creative Commons Attribution NonCommercial 3.0 License (CC BYNC 3.0).

(C) Copyright A. Jain, 2014

Licensee PAGEPress, Italy

Rheumatology Reports 2014; 6:5450

doi:10.4081/rr.2014.5450

sistent with scleroderma. Ultrasound abdomen and chest revealed bilateral pleural effusion with pericardial effusion and normal liver, spleen. Kidney size was normal and there was no free fluid in the abdomen. Chest X-ray revealed massive cardiomegaly with normal lung fields (Figure 2). ECG was suggestive of low voltage complexes and 2D-echocardiography revealed the presence of pericardial effusion, $11 \mathrm{~mm}$ anterior to right ventricle, $15 \mathrm{~mm}$ posterior to the left ventricle, $26 \mathrm{~mm}$ lateral to the left ventricle with the diastolic collapse of right ventricle (Figure 3). Patient was diagnosed as a case of mixed connective tissue disorder with pericardial tamponade and pancytopenia. Patient was hemodynamically stable and therefore she was planned for conservative treatment. Patient was immediately started on oral prednisolone in a dose of 1 $\mathrm{mg} / \mathrm{kg} /$ day $(50 \mathrm{mg} /$ day) along with levothyroxine in dose of $50 \mathrm{mcg} /$ day. Patient's improved over the next 7 days with improvement of blood counts and she was continued on same dose of glucocorticoids for another 3 weeks. Patients blood investigations revealed resolution of pancytopenia with complete normalization of total leucocyte and platelet counts. Patient's swelling over the face and legs reduced and repeat urine examination showed 24 hour protein of $350 \mathrm{mg} / 24$ hours and echocardiography done at the end of 4 weeks showed resolution of tamponade effect with only minimal pericardial effusion remaining. Patient was discharged from the hospital after 4 weeks of prednisolone treatment and was also started on mycophenolate mofetil at the time of hospital discharge. Cyclophosphamide was not 
added due to the risk of infertility and mycophenolate was added only after the improvement of blood counts. Patient was followed up in the OPD with gradual tapering of the prednisolone dose.

\section{Discussion}

Mixed connective tissue disorder was first described by Sharp et al. in 1972 as an overlap syndrome with features of SLE, systemic sclerosis and polymyositis along with the presence of high titers of anti-U1RNP antibodies. ${ }^{1}$ Since then, MCTD has been characterized in a better way and is known to evolve from one subtype into another. A number of autoantibodies are reported in MCTD which includes anti-U1 RNP (IgG subtype), anti-ACE2, anti-cardiolipin, antiendothelial cell antibody, and others including anti-Ro/SS-A, anti-Sm, anti-ssDNA, anti-dsDNA, although not specific for MCTD. ${ }^{2}$ Cardiac involvement in MCTD has been reported widely from $11-85 \%$ depending on the method used for detection..$^{34}$ Alpert et al. reported a number of cardiac abnormalities in MCTD including pericarditis (with or without effusions, 29\%), mitral valve prolapse (10\%), cardiac conduction abnormalities, myocarditis, intimal hyperplasia of the intramural coronary arteries, perivascular leucocytic infiltrates and pulmonary artery hypertension. ${ }^{4}$ Pericarditis is the commonest cardiac manifestation of MCTD variably reported to occur in $29 \%, 43 \%, 56 \%$ of the cases, detected either ante-mortem or by autopsy. ${ }^{4-6}$ Pericardial effusions are found in $24-38 \%$ of the patients by echocardiography, ${ }^{4,7}$ Interestingly, pericardial

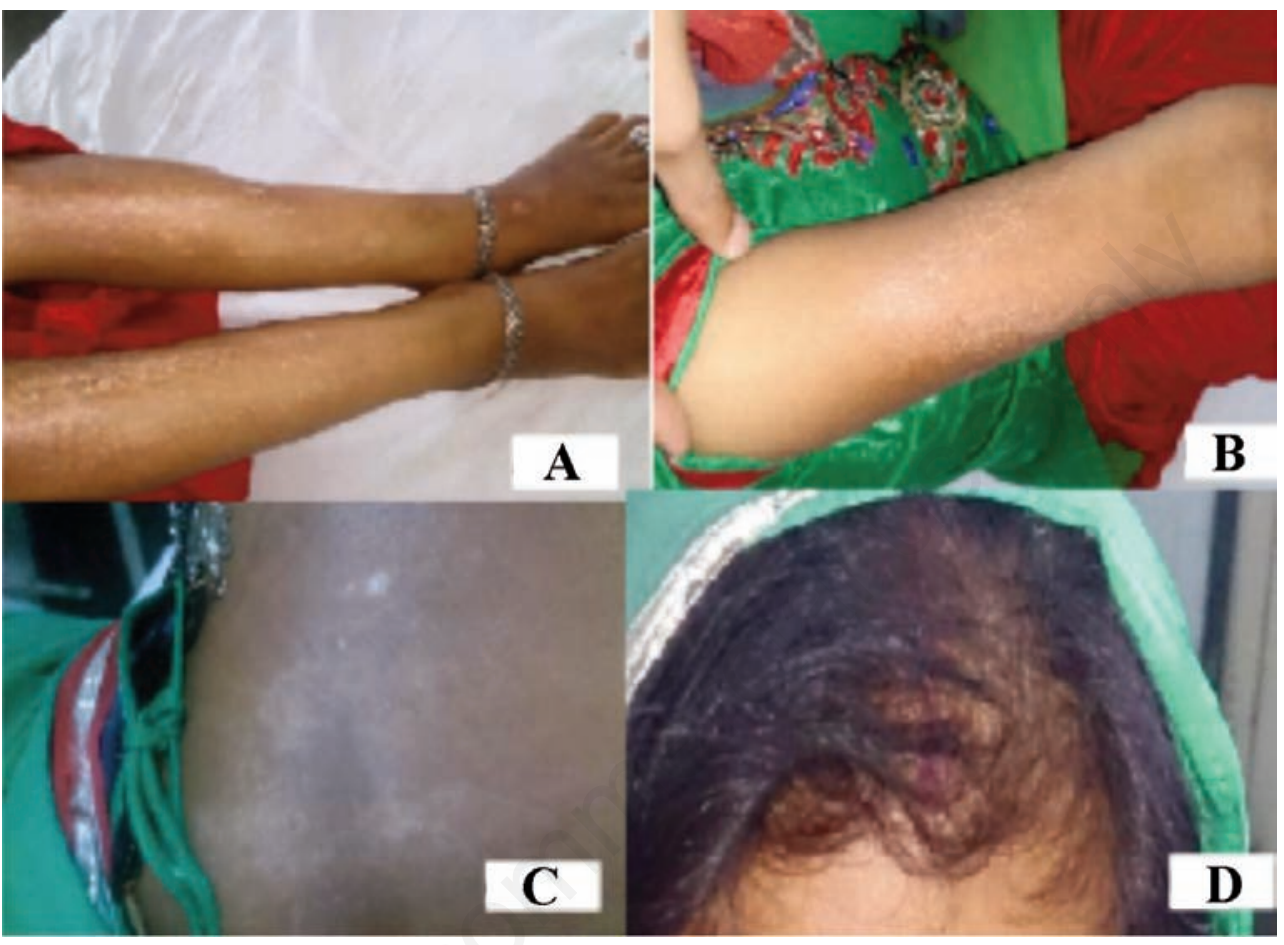

Figure 1. Clinical pictures of the patient with MCTD showing thickened skin of bilateral lower limbs (A), cubital fossa along with perifollicular hypopigmented macules (B), nape of the neck (C), and non-scarring alopecia (D).

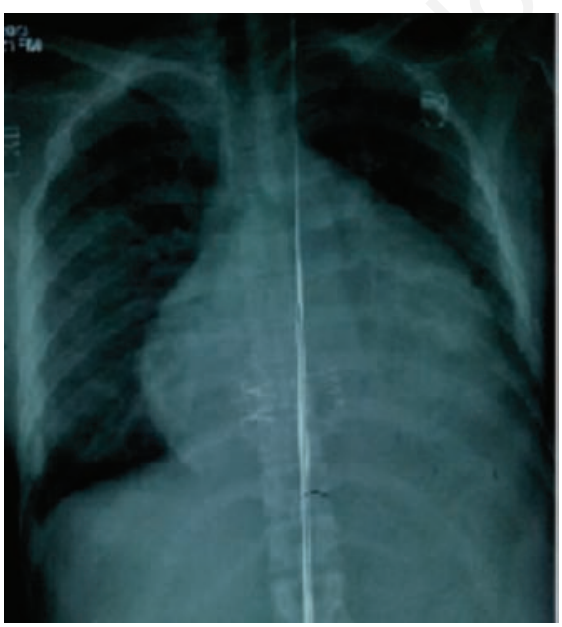

Figure 2. Chest X-ray of the patient showing massive cardiomegaly due to a large pericardial effusion.
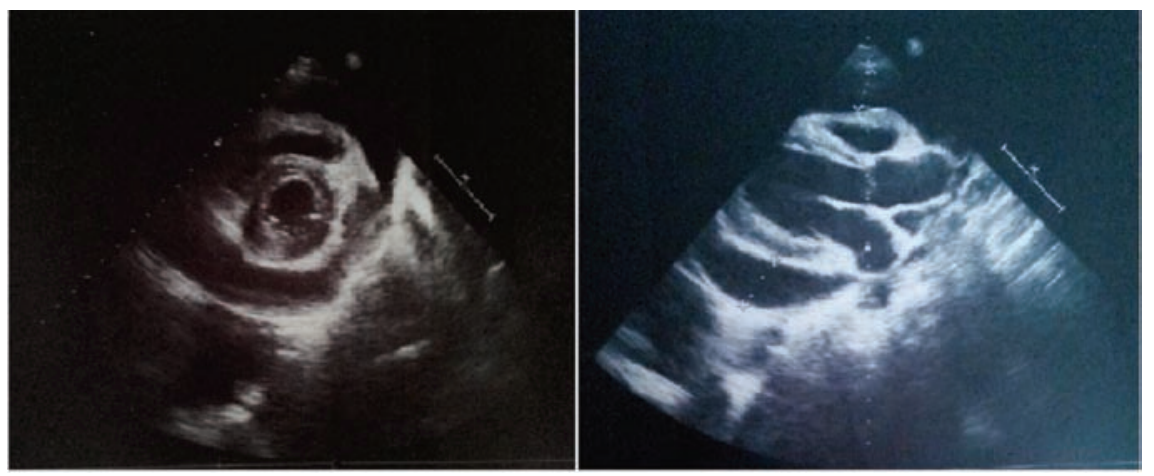

Figure 3. Picture of the 2D echocardiograph of the patient showing large pericardial effusion surrounding the heart. 
involvement in MCTD is usually asymptomatic and clinically significant disease is present in only $10 \%$ of cases. Even rarer in MCTD is tamponade which has been reported in only 6 cases till date, usually in the setting of an established disease. Pericardial tamponade can present acutely with sudden deterioration of the condition or sub-acutely with gradually progressive features. Pathogenesis of pericarditis in MCTD is unknown but has been proposed to be complement mediated. ${ }^{8}$ Treatment of pericardial disease in MCTD is not established however, literature provides evidence of effectiveness of NSAIDS, colchicines, corticosteroids, and immunosuppressant in the management of pericarditis associated with MCTD. ${ }^{9}$ Complete resolution of pericardial effusions has been reported with the use of corticosteroids. Hematological manifestations in MCTD includes anemia of chronic disease, leucopenia, coomb's positive hemolytic anemia, ${ }^{10}$ pure red cell aplasia. ${ }^{11}$ Thrombocytopenia has been described in MCTD and causes can be IgG antiplatelet antibody mediated platelet destruction, ${ }^{12}$ thrombotic thrombocytopenic purpura, ${ }^{13}$ or antiphospholipid antibody syndrome. Antiphospholipid antibodies have been seen in $15 \%$ of the patients with MCTD (different from anti-b2GP1) and are associated with pulmonary artery hypertension but are usually not associated with thrombotic eventsalthough,,14 case reports of sinus vein thrombosis due to antiphospholipid antibody syndrome in MCTD have been described in the literature. ${ }^{15}$

Pancytopenia is a very rare finding in MCTD and is even rarer as a first manifestation. Kumar et al. ${ }^{9}$ reported a case of pericardial tamponade in MCTD along with review of the 5 previously reported cases, making a total of 6 reported cases till date. Pericardial tamponade is reported in only 1 case as a first presentation of the disease, 4 cases required invasive procedure (pericardiocentesis or pericardiostomy) in combination with corticosteroids for the treatment, 1 case was managed with only corticosteroids and 1 was treated successfully with only NSAIDS. None of the 6 reported cases had pancytopenia at presentation or at any point of time. Our patient presented with pericardial tamponade and pancytopenia was detected on investigations. Diagnosis of MCTD was made by criteria proposed by Kasukawa et al. ${ }^{6}$ Patient was treated with high dose corticosteroids for 1 month with complete normalization of blood counts and resolution of pericardial effusion. We probably report the first case of pericardial tamponade with pancytopenia as the first presentation of MCTD, which completely recovered with only corticosteroids, without any invasive intervention.

\section{Conclusions}

Mixed connective tissue disorder is an overlap syndrome of scleroderma, SLE and polymyositis. Pericardial tamponade although rare, has been reported in this disorder and represents a potentially fatal complication. Present case highlights the fact that cardiac tamponade can be the initial presentation of the MCTD. Prompt recognition of the underlying MCTD as the cause of pericardial tamponade is a key in the management and timely treatment with corticosteroids can effectively reverse this fatal condition. Similarly, pancytopenia is a rare finding in MCTD and presence of pancytopenia in a patient with pericardial tamponade should make a clinician to direct his diagnosis towards mixed connective tissue disorder under appropriate clinical scenario.

\section{References}

1. Sharp GC, Irvin WS, Tan EM, et al. Mixed connective tissue disease, an apparently distinct rheumatic disease syndrome associated with a specific antibody to an extractable nuclear antigen (ENA). Am J Med 1972;52:148-59.

2. Ortega-Hernandez 0, Shoenfeld Y. Mixed connective tissue disease: an overview of clinical manifestations, diagnosis and treatment. Best Pract Res Clin Rheumatol 2012;26:61-72.

3. Lundberg I, Hedfors E. Clinical course of patients with anti-RNP antibodies. A prospective study of 32 patients. J Rheumatol 1991;18:1511-9.
4. Alpert MA, Goldberg SH, Singsen BH, et al. Cardiovascular manifestations of mixed connective tissue disease in adults. Circulation 1983;68:1182-93.

5. Singsen BH, Bernstein BH, Kornreich HK, et al. Mixed connective tissue disease in childhood: a clinical and serologic survey. J Pediatr 1977;90:893-900.

6. Kasukawa R. Mixed connective tissue disease. Intern Med 1999;38:386-93.

7. Oetgen WJ, Mutter ML, Lawless OJ, Davia JE. Cardiac abnormalities in mixed connective tissue disease. Chest 1983;83: 185-8.

8. Langley RL, Treadwell EL. Cardiac tamponade and pericardial disorders in connective tissue diseases: case report and literature review. J Natl Med Assoc 1994;86: 149-53.

9. Kumar MS, Smith M, Pischel KD. Case report and review of cardiac tamponade in mixed connective tissue disease. Arthritis Rheum 2006;55:826-30.

10. Sullivan WD, Hurst DJ, Harmon CE, et al. A prospective evaluation emphasizing pulmonary involvement in patients with mixed connective tissue disease. Medicine 1984;63:92-107.

11. Julkunen H, Jantti J, Pettersson T. Pure red cell aplasia in mixed connective tissue disease. J Rheumatol 1989;16:1385-6.

12. Shimizu Y, Nojima Y, Mori M. Increased levels of platelet-associated immunoglobin $\mathrm{G}$ in a patient with mixed connective tissue disease. Hong Kong Med J 2002; 8:285-7.

13. Ter Borg EJ, Houtman PM, Kallenberg CG, et al. Thrombocytopenia and hemolytic anemia in a patient with mixed connective tissue disease due to thrombotic thrombocytopenic purpura. J Rheumatol 1988;15: 1174-7.

14. Mendonca LL, Amengual 0, Atsumi T, et al. Most anticardiolipin antibodies in mixed connective tissue disease are beta2-glycoprotein independent. J Rheumatol 1998; 25:189-90.

15. Gluck T, Muller-Ladner U, Speicher A, et al. Fatal sinus vein thrombosis in a patient with mixed connective tissue disease and secondary antiphospholipid antibody syndrome. Med Klin 2001;96:361-4. 